\title{
Two case reports on Mandibular metastases
}

\author{
Adesina O. Adewale ${ }^{1}$, Ladeji A. Mofoluwake ${ }^{2}$, Opaleye T. Olamide ${ }^{1}$ and Salami A. Yussuf ${ }^{1}$ \\ Ghana Med J 2018; 52(3): 168-172 doi: http://dx.doi.org/10.4314/gmj.v52i3.11
}

\author{
${ }^{1}$ Department of Oral \& Maxillofacial Surgery, Faculty of Dentistry, Lagos State University College of Medicine, \\ Lagos State, Nigeria. \\ ${ }^{2}$ Department of Oral Pathology, Faculty of Dentistry, Lagos State University College of Medicine, Lagos State, \\ Nigeria
}

Corresponding author: Dr. Adesina O. Adewale

E-mail: femmyadesina2000@yahoo.com

Conflict of interest: None declared

\section{SUMMARY}

Metastatic tumours of the oral cavity are not common. The most common site for bone metastases in the head and neck region is the mandible. Metastatic lesion of the mandible most commonly originate from the lungs in men and breast in women, and these lesions (or tumours) usually are carcinomas rather than sarcomas. We report two cases of metastatic lesions in the mandible: (1) A 19year old male with right mandibular swelling and a nodular swelling in the distal two-third of the right tibia. Radiograghs revealed sclerotic lesion with a sunburst appearance in both the jaw and tibia. Histology of the jaw lesion confirmed a metastatic osteosarcoma of the mandible. (2) A 51year old female with a right mandibular swelling with associated swelling in the anterior neck, right shoulder and right parietal region of the skull. Computerised tomography scan (CT) of the jaws showed an osteolytic lesion of the right mandible. CT scan of the brain also showed a large lytic lesion seen in the posterior aspect of the right parietal bone. Histology of the jaw mass confirmed a metastatic follicular carcinoma from the thyroid. Mandibular metastasis may be the first presenting feature of underlying occult malignancy of another primary site. Therefore, clinicians should maintain a high level of suspicion while evaluating patients with a history of cancer presenting with oral lesions.

Keywords: metastatic tumours, mandible, tibia, thyroid

\section{INTRODUCTION}

Metastatic tumours of the oral cavity are not common. They represent only about $1 \%$ of oral tumours. They affect the jaws much more frequently than soft tissues. ${ }^{1}$ The most common site for bone metastases in the head and neck region is the mandible (especially the angle, premolar and condylar part), comprising over $70 \%$ of all malignant metastatic tumours in the oral cavity. ${ }^{2}$ Females are more affected than males ${ }^{3}{ }^{3}$ The most common primary tumour sites for jaw metastases in women are breast, adrenals, genital organs, and thyroid. Meanwhile, the most frequent sites of primary tumours in men are lungs, prostate, kidney, bone, and adrenals. . $^{3,4}$

Metastatic involvement of the mandible and maxilla cause a variety of signs and symptoms. These includes swelling, painful gum, loose or extruded teeth, exophytic growth in soft tissue, trismus, pathologic fractures and numbness or paraesthesia of the lower lip and chin. ${ }^{5,6,7}$ In some cases, patients may be asymptomatic, in which case the diagnosis is made as a radiological finding. ${ }^{8}$
Diagnosis of metastatic jaw bone lesions is challenging because of their low prevalence. In addition, many of the bone lesions are at the center of the bone and cannot be detected by physical examination. These lesions can be mistaken with other infectious or inflammatory diseases of the jawbone and adjacent structures because of clinical and radiographical similarities. ${ }^{9,10}$ The mechanism by which tumours can spread to the oral cavity is poorly understood. ${ }^{11}$ In this report we present two cases of metastases to the mandible, the clinical manifestations and the attendant difficulty in diagnosis.

\section{CASE REPORTS}

\section{Case Report 1}

A 19-year-old male patient who had reported at the orthopaedic clinic in the same hospital with a year history of right leg swelling was referred to the maxillofacial clinic for an assessment of a right lower jaw swelling. He had noticed the swelling two months previously which has gradually increased to present size (Figure 1). 
The patient had presented to the orthopaedic clinic where a general evaluation and biopsy of the leg swelling had been taken. The biopsy report revealed features consistent with osteosarcoma of the tibia.

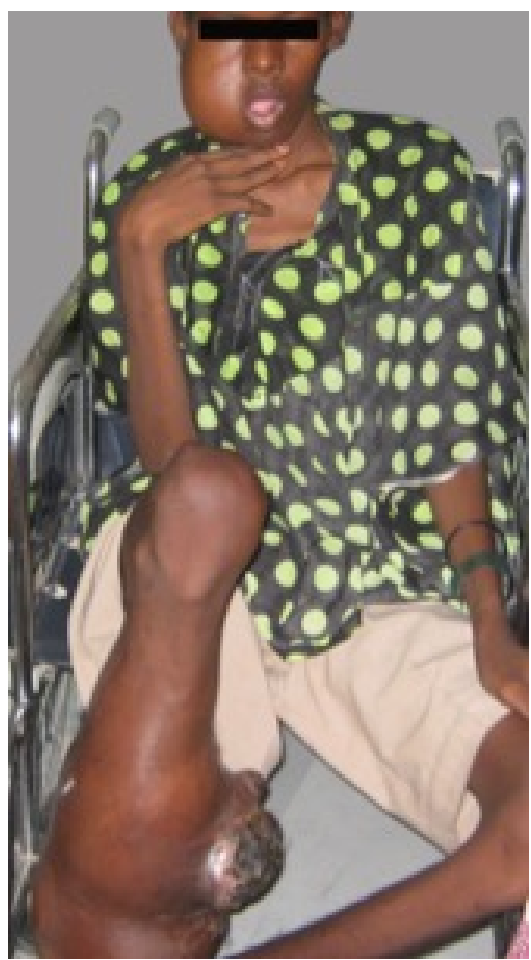

Figure 1: Case 1 showing the right mandibular lesion and the lesion on the swollen right ankle

General examination revealed a large nodular swelling on the distal two -third of the tibia. There was no abnormal mobility felt in the distal end of right tibia. There was a necrotic ulcer in the region of the medial ankle.

On extraoral examination, a diffuse swelling was seen on the right mandibular region involving the right body and angle of the mandible, measuring approximately $8 \times 6 \mathrm{~cm}$ extending superiorly $1 \mathrm{~cm}$ below the level of outer canthus of the eye and inferiorly $1 \mathrm{~cm}$ above the lower border of the mandible, anteriorly from the corner of the mouth to $1 \mathrm{~cm}$ in front of the tragus of ear.

The right sub-mandibular lymph nodes were palpable, which were non-tender, mobile, firm, round to ovoid in shape, measuring approximately $1 \mathrm{~cm} \times 1 \mathrm{~cm}$. Intraoral examination revealed a pedunculated mass of approximately $3 \times 2 \mathrm{~cm}$ arising from right retromolar region, causing obliteration of the buccal vestibule from the lower right canine to the lower right third molar. The lesion intraorally was pink in colour; smooth-surfaced with areas of indentation of teeth on superior surface, mildly tender, and firm in consistency.
There was mobility of the related teeth, and the lower right third molar was found missing. Posterior-anterior view of the skull shows sclerotic lesion extending from the region of right canine to the right ramus also involving the right condyle. It also revealed a sunburst appearance from the canine region to the angle of the mandible. $\mathrm{PA}$ view of the right tibia also showed a large sclerotic lesion, with soft tissue permeation in the lower two-third of tibia and extension of the lesion through the periosteum resulting in a sunburst appearance.

Laboratory Investigations including Full blood count, liver function tests (LFT), and serum chemistry profiles were all normal. Chest X-ray was negative for any signs of metastasis. Based on the clinical and radiographic findings, a provisional diagnosis of a metastatic osteosarcoma of the mandible was considered.

An incisional biopsy was performed. Histological examination revealed a fibro-osseus tissue, within which were atypical osteoblasts-like cells laying down osteoid. Also seen were bony trabeculae, endothelial lined vascular channels and areas of haemorrhage.

The patient died following amputation of the right leg. The cause of death was from cardiac failure following uncontrolled haemorrhage in the immediate post -operative period.

\section{Case Report 2}

A 51year old female trader, presented at the Oral and Maxillofacial Surgery Department of the Lagos State University Teaching Hospitals, with a right mandibular swelling of about one-year duration and anterior neck swelling of about 16years duration (Figures 2). She had previously presented to the general surgical department of the same hospital and an assessment of the neck mass had been made. She had a thyroidectomy done and the histopathological report of the surgical specimen revealed a well differentiated follicular thyroid carcinoma. She is a known hypertensive patient on Lisinopril and Amlodipine.

Extraoral examination revealed obvious facial asymmetry due to a right mandibular swelling with associated swelling in the anterior neck, right shoulder and right parietal regions. The mandibular swelling measured approximately $8 \mathrm{~cm} \times 7 \mathrm{~cm}$. It extended superiorly from the zygoma to the right tragus and inferiorly from the right canine region to the angle of the mandible. The overlying skin appeared normal, swelling is firm in consistency, not tender or warm to touch. Intraoral examination revealed a swelling which extend from the right canine to the right retro-molar region. 
Overlying mucosa appeared clinically healthy, but there was mobility of the premolar and the molar teeth in relation to the swelling.

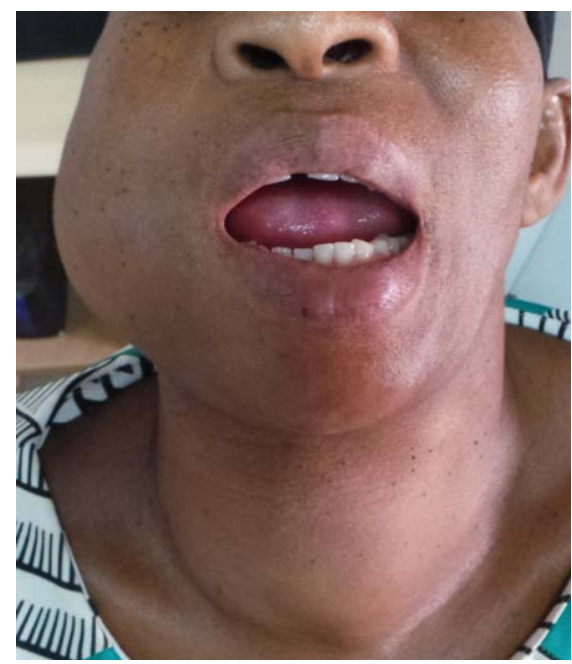

Figure 2: Metastatic carcinoma of the right mandible from the thyroid gland.

Computerised tomography scan of the jaws showed an osteolytic lesion of the right mandible from the region of right canine to the angle. Computerised tomography scan of the brain also showed a large lytic lesion in the posterior aspect of the right parietal bone with soft tissue component mildly compressing the adjacent brain parenchyma. Chest X-ray showed nodular soft tissue dense opacities of varying sizes in both lung fields, worse in the lower zones. There was an expansile lytic lesion in the right acromion. Laboratory investigation showed normal values of T3, T4 and TSH. Other parameters including Full blood count, liver function tests (LFT), and serum chemistry profiles were all normal. Based on the clinical and radiographic findings, a provisional diagnosis of metastatic carcinoma to the right mandible from the thyroid gland was considered.

Incisional biopsy of the mandibular mass was done. Histopathology revealed a piece of tissue composed of numerous colloid-filled spaces lined by single layer of follicular cells in areas. Also seen in other areas was a distortion of glandular architecture with cells appearing atypical. A diagnosis of Secondary Follicular thyroid carcinoma was made. She was referred to the Oncology Department for palliative radiotherapy and chemotherapy.

\section{DISCUSSION}

Mandibular metastasis may be the first presenting feature of underlying occult malignancy of another primary site. ${ }^{13}$ Metastatic lesion of the mandible most commonly originate from the lungs in men and breast in women ${ }^{14}$, and these lesions (or tumours) usually are carcinomas rather than sarcomas. ${ }^{15}$ In about $30 \%$ of cases of patients with jaw bone metastases, the primary tumour is asymptomatic and not diagnosed. ${ }^{16}$ However, in the two cases reported the primary lesions were the main reason why the patients reported at the hospital.

Secondary deposits from primary growth to a distant organ occur mainly by invasion of blood vessels, or through lymphatic or tissue planes. However, many of the invading malignant cells gaining access to the mandible is through the blood vessels. ${ }^{17}$ Although the mechanism for blood-borne metastases to the head and neck area is poorly understood, one possible route may be through the Batson's plexus which is a valveless vertebra venous plexus. ${ }^{11}$ This might allow a retrograde spread of tumour cells by passing infiltration through the lungs. In the two cases presented we observed that the metastatic lesions were one sided. However, the reason for this is not clear. The commonest site involved as reported by most authors is the premolar - molar region. This may be due to the abundant cancellous bone in this region and its proximity to the main branches of the inferior alveolar artery, which explains the high tendency of haematogenic spread. ${ }^{18}$ However, some authorities believed it is due to angulation of the inferior alveolar vessels leading to slowing down of the blood stream and fall out of the circulating metastatic cells around these regions. ${ }^{19}$

Metastatic tumour of the mandible may sometimes give a vague presentation or entirely asymptomatic, which may sometimes be overlooked or found during radiographic survey of the bone. However, most often it presents a jaw mass, jaw pain, toothache or tooth mobility, pathologic fracture of the mandible or neurologic symptoms due to involvement of the inferior alveolar nerve resulting in paraesthesia or numbness. ${ }^{20}$

Clausen and Poulsen, however, described the criteria for diagnosing metastatic lesions as: proved primary tumour with histopathologic confirmation and radiographic evidence, mandibular metastasis with histopathologic evidence and radiographic evidence, histopathologic correlation of the metastatic lesion with the primary site. In the event of close proximity to the primary site there must be a wide margin around the primary site to rule out direct extension from the primary site ${ }^{18}$. In the two cases reported there were similarity in the histopathologic presentation of both the primary and the metastatic lesions.

The radiographic presentation in metastatic tumours is non-specific as a result many clinicians have erroneously diagnosed various types of cysts, benign lesions or even as lesions of infective or traumatic origin. 
Metastatic lesions often may present as osteolytic (bonedestroying) or osteoblastic(bone-forming). ${ }^{19}$ The typical radiographic sunburst appearance which is frequently associated with osteosarcoma was observed in the radiographic views of both the right mandible and the right tibia in the first case reported. This, however, may show similarity in the aggressive nature of both the primary and the metastatic sites.

The treatment and prognosis are varied, depending on the site of the primary lesion and the degree of metastatic spread. Further work-up to identify the primary site and estimate the stage and grade of metastatic involvement may be necessary. ${ }^{21}$ If the patient is in a terminal stage of disease and there is widespread metastasis, relief of symptoms can often be obtained with radiation or chemotherapy. Surgical treatment only is used in cases, where no other metastases are demonstrated. ${ }^{22}$ When the oral lesion is found to be the only metastatic lesion, resection seemed to result in improved prognosis as indicated in the first case presented.

Prognosis of oral metastatic osteosarcomas, however, is very poor with the mean time from the appearance of metastasis to death being 7.3 months. ${ }^{23}$ Generalized skeletal metastases are usually an ominous event and are treated palliatively ${ }^{24} \cdot{ }^{24} \mathrm{~A}$ palliative treatment was the option for our second patient due to the widespread metastasis. The prognosis for patient with metastatic carcinoma of the jaws is grave, with a $10 \%$ five-year survival and more than two thirds of the patient die within a year. ${ }^{25}$

\section{CONCLUSION}

Mandibular metastasis may be the first presenting feature of underlying occult malignancy of another primary site. Diagnosis of metastatic jaw bone lesions is challenging because of their low prevalence and often because they are mistaken for other lesions. Therefore, clinicians should maintain a high level of suspicion while evaluating patients with a history of cancer presenting with oral lesions.

\section{REFERENCES}

1. Piattelli A, Fioroni M. Gingival metastasis from a prostate adenocarcinoma: Report of a case. J Periodontal 1999; 70(4):441-4.

2. Piattelli A, Fioroni M. Gingival metastasis from a medullary thyroid carcinoma: Case report. J Periodontal 2000;7: 112-6. .

3. Cawson RA, Lucas's. Pathology of tumors of the oral tissues.5e, $5^{\text {th }}$ ed. USA: Churchill Livingstone 1998. p. 425-7.

4. Hirshberg A, Leibovich P, Buchner A. Metastatic tumors to the jaw bone: Analysis of 390 cases. J Oral Pathol Med1994;23:337-41.
5. D’Silva NJ, Summerlin DJ, Cordell KG, Abdelsayed RA, Tomich CE, Hanks CT, et al. Metastatic tumors in the jaws : A retrospective study of 114 cases. $J \mathrm{Am}$ Dent Assoc.2006;137(12):1667-72.

6. Akinbami B. Metastatic carcinoma of the jaws: A review of literature. Nigeria. J Med. 2009;18(2):13942.

7. Ryba F, Rice S, Hutchison I. Numb chin syndrome: an ominous clinical sign. $\mathrm{Br}$ Dent $J$. 2010;208(7):283-5.

8. Antonio AA ,Antonio PA;Gnathic bone metastasis:A retrospective study of 10 cases. Rev. Bras. Otorrinolaringol. 2008.74(4) Pg.561-565.

9. Ög ütcen-Toller M, Metin M, Yıldız L. Metastatic breast carcinoma mimicking periodontal disease on radiographs. J Clin Periodontol. 2002;29(3):269-71.

10. Selden HS, Manhoff DT, Hatges NA, Christoph Michel R. Metastatic carcinoma to the mandible that mimicked pulpal/periodontal disease. $J$ Endodont. 1998;24(4):267-70.

11. Neville BW. Oral \& maxillofacial pathology. 2nd ed. USA: W.B. Saunders; 2002. p. 582-3.

12. Hirshberg A, BergerR, Allon I,Kaplan I .Metastatic Tumors to the Jaws and Mouth . Head Neck Pathol 2014 Dec; 8(4): 463-474

13. Saha K, Jash D, Maji A. Mandibular metastasis with pulmonary cannon balls: Presentation of follicular carcinoma thyroid. Med J DY Patil Univ [serial online] 2016 [cited 2017 Aug 9];9:234-6

14. Hirshberg A, Buchner A. Metastatic tumors to oral region an overview. Eur J Cancer B Oral Oncol. 1995;31B:355-60.

15. Keller EE, Gunderson LL. Bone disease metastatic to the jaws. J Am Dent Assoc. 1987;115:697-701.

16. Bodner L, Geffen DB. Metastatic tumor of the jawdiagnosis and management. (81).Refuat Hapeh Vehashinayim. 2003;20:59-61.

17. Moore GE, Sandberg AA, Watne AL. The Comparative Size and Structure of Tumor Cells and Clumps in the Blood, Bone Marrow, and Tumor Imprints, Cancer 1960;13: 111-117.

18. Blackwood, HJJ. Metastatic carcinoma of the mandibular condyIe. Oral Surg.1956 Dec.9(12) 1318132319. Pool EH, Dunlop GR. Cancer Cells in Blood Stream .Am. J. Cancer 1934.;21;99-102

19. Glaser C, Lang S. Clinical manifestations and diagnostic approach to metastatic cancer of the mandible Int. J. Oral Maxillofac. Surg, 1997,26; pp. 365-368

20. Clausen F, PoulsenH. Metastatic Carcinoma to the jaws. Acta Pathol. Microbiol. Scand. 1963;57:361.

21. 22. Catone GA, Henny FA. Metastatic gastric adenocarcinoma of the mandible. $J$ Oral Surg 1969;27(1):36-4023.

22. Rodrigues G. Metastatic osteosarcoma to the maxilla. Kuwait Med J. 2003;25:219-21. 


\section{Case Report}

23. Soussan I, Mohammad M, Ali L. Metastasis of a gastric adenocarcinoma to the mandible Oral Oncology EXTRA 200440 85-87
24. Regezi JA, Sciubba JJ. Oral pathology clinical pathologic correlations. 5th ed. Philadelphia: W.B. Saunders; 2003 p 413-5 